\title{
THE PRESENCE OF SUGAR IN THE URINE OF NEW BORN INFANTS BEFORE THE INTAKE OF FOODS *
}

\section{FRANCES MILLIKIN}

CHJCAGO

Czerny and Keller ${ }^{1}$ mention that Mesnil found sugar occasionally in the urine of infants during the first few days of life, and Nothman ${ }^{2}$ found sugar in the urine of premature infants. The presence of sugar was shown by reduction tests, and in the case of Nothman the osazone was prepared which indicated the presence of lactose. By using the Benedict-Osterberg ${ }^{3}$ method, Greenthal ${ }^{4}$ showed that the urine of all infants contains sugar. This would be expected from the results obtained by Benedict, Osterberg and Neuwirth. ${ }^{5}$ These authors found determinable quantities of sugar at all times, both in the urine of normal dogs, and those fasting for four-day periods, and in the urine of adults.

Thanks to the kindness of Dr. Sauer, we were able to obtain the urine of a series of new born infants for sugar determination.

The urine was collected during the period preceding the first feeding. None of the births was difficult, so that the results cannot be vitiated by the transitory traumatic glycosuria described by Hoeniger. ${ }^{6}$ The method employed for the determination was that described by Benedict and Osterberg, ${ }^{3}$ which has the important advantage of eliminating the possibility of the interference, in the determinations, of albumin, which occurs in the urine of the newly born. In all instances, duplicate determinations were made, all of which agreed very closely. In every case the urine was strongly acid to litmus. The first five cases are not recorded in the tables. They showed 0.036, 0.034, 0.072, 0.05 and 0.136 per cent. sugar, respectively.

Table 1 shows the quantitative determinations of sugar in the urine of individual infants. In Table 2, combined specimens from two or three infants, as indicated, were used. The amounts of urine per individual varied between 6 and 34 c.c. The amonts of total urinary

\footnotetext{
* Received for publication, Feb. 5, 1921.

* From the Otho S. A. Sprague Memorial Institute of the Children's Memorial Hospital.

1. Czerny and Keller: Des Kindes Ernährung, etc.

2. Monatschr. f. Kinderh. 8:377, 1909.

3. Benedict and Osterberg: J. Biol. Chem. 34: 195 (April) 1915.

4. Greenthal: Am. J. Dis. Child. 20:556 (Dec.) 1920.

5. Benedict, Osterberg and Neuwirth: J. Biol. Chem. 34: 217 (April) 1915.

6. Hoeniger: Deutsch. med. Wchnschr., 1: 5001911.
} 
sugar for the collection period ranged from 1.722 to $27.948 \mathrm{mg}$, with an average of $9.536 \mathrm{mg}$. The concentration of sugar in Tables 1 and 2 varied between 0.027 and 0.166 per cent., with an average of 0.078 per cent. Too much importance should not be attached to averages for so few cases.

Benedict, Osterberg and Neuwirth ${ }^{5}$ found sugar in the urine of adults collected for two hour periods in the morning before food was taken, in concentrations of from 0.04 to 0.16 per cent. for one subject and from 0.03 to 0.049 per cent. for another. Greenthal ${ }^{*}$ showed sugar to be present in the urine collected for eight hour periods from infants on ordinary diets in concentrations of from 0.045 to 0.092 per cent. The twenty-four hour urine of a normal adult on ordinary diet contained from 0.042 to 0.22 per cent sugar, that of another from 0.04 to 0.126 per cent.

TABLE 1.-Quantitative Determination of Sugar in Urine of Infants

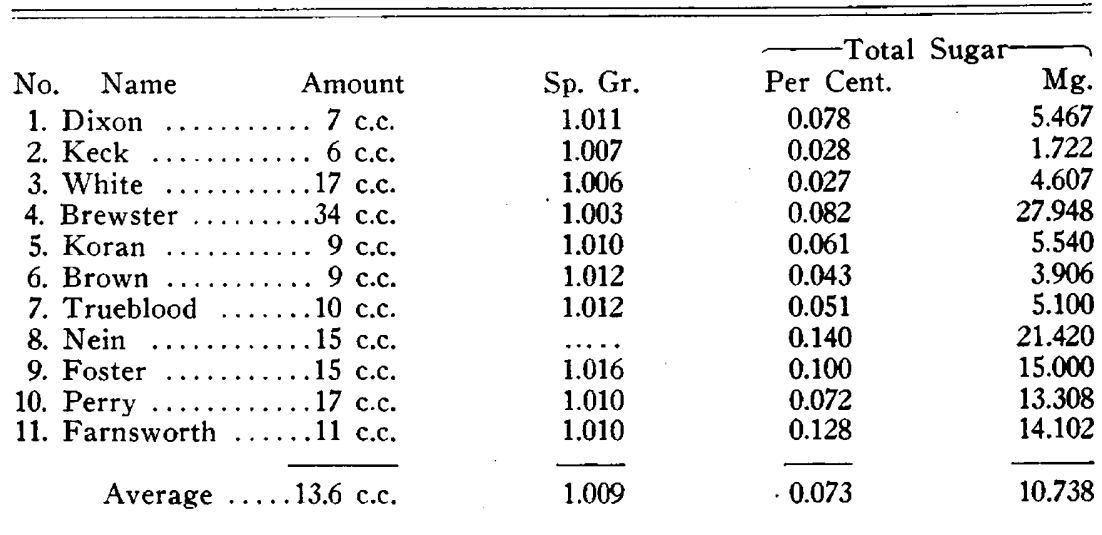

In Table 3 two determinations of the unfermentable sugar are given. There is sufficient reason to accept the fermentable sugar as glucose, but the nature of the unfermentable sugar is not known. In this table the unfermentable sugar was present in lower percentages (12.6 and 30.8 per cent.) than in the urine of infants and adults on regular diets or in the urine of adults during the fasting period of the day. For example, in urine collected from adults during the two-hour period in the morning before food was taken, Benedict, Osterberg and Neuwirth found unfermentable sugar to constitute from 57 to 73 per cent. (at one time falling as low as 36.3 per cent.) of the total urinary sugar for one subject and from 41.8 to 71.0 per cent. for the other. For adults on mixed diets they found the twenty-four hour urine 
specimens to contain unfermentable sugar constituting from 40 to 65 per cent. of the total urinary sugar. Greenthal ${ }^{4}$ demonstrated that of the total urinary sugar of infants on ordinary diet, from 38 to 49 per cent. was unfermentable sugar.

TABLE 2.-Quantitative Determination of Sugar in Combined SPECIMENS OF URINE

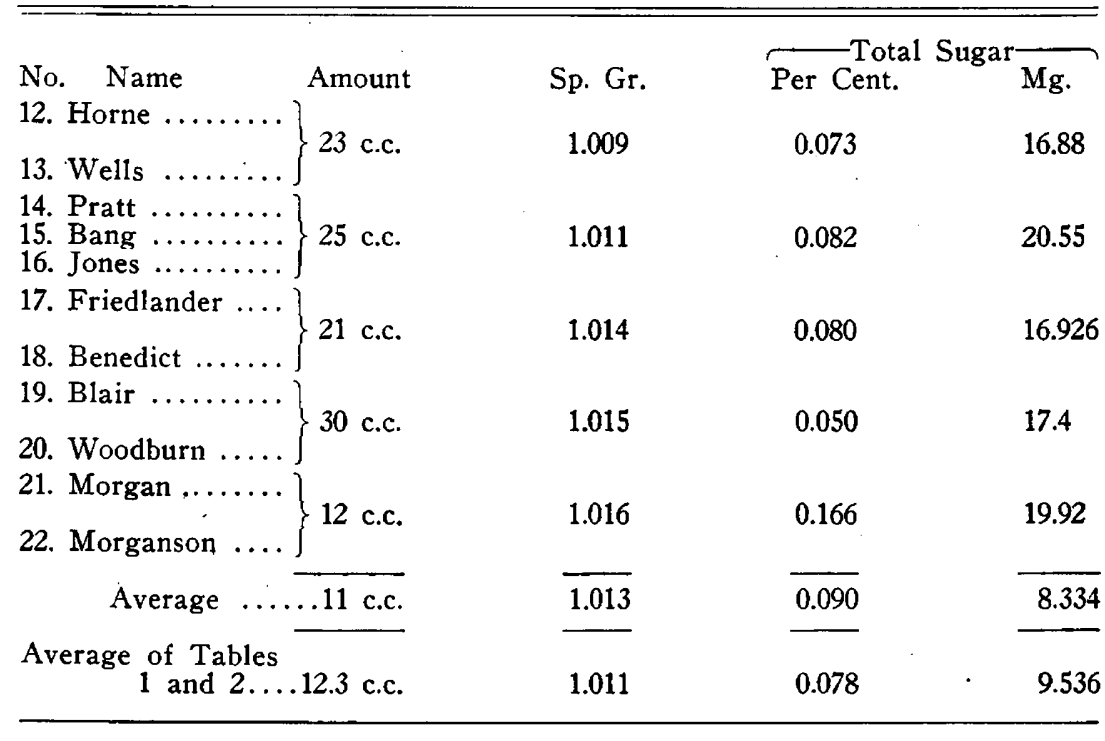

The results of this series of urinary sugar determinations show that sugar does constantly occur in the urine of newly born infants before food is taken, and that it is present in about the same concentrations as in the urine of normal adults before food is taken in the morning.

TABLE 3.-Determinations of Unfermentable Sugar

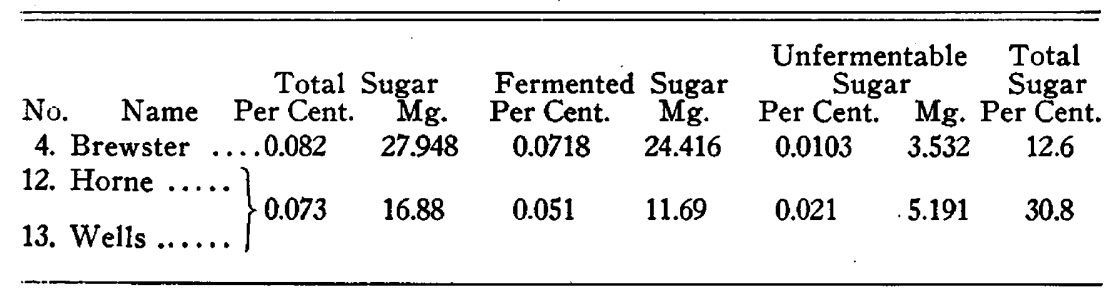

The range of concentrations can easily explain why sugar is sometimes found in the urine of the newly born by the usual copper reduction tests. For instance, Miss Felcher, working with Dr. Woodyatt,

7. With the permission of Dr. Woodyatt these data are published. 
found that Haines' test gives a positive reaction readily with a concentration of sugar in the urine exceeding 0.07 per cent when 5 c.c. of the reagent and 2 c.c. urine are used.

As in the case of adults, as demonstrated by Benedict, Osterberg and Neuwirth, and of infants, as shown by Greenthal, this urinary sugar can be separated into a fermentable and a nonfermentable portion. 